\title{
Cryorecanalization: A new approach for the immediate management of acute airway obstruction
}

\author{
Martin Hetzel, MD \\ Juergen Hetzel, MD \\ Christian Schumann, MD \\ Nikolaus Marx, MD \\ Alexander Babiak, MD
}

From the Department of Internal Medicine II-Pulmonary Medicine, University Medical Center Ulm, Ulm, Germany.

Received for publication Oct 22, 2003; revisions requested Dec 15, 2003; accepted for publication Jan 22, 2004.

Address for reprints: Martin Hetzel, MD, Department of Internal Medicine II-Pulmonary Medicine, University Medical Center Ulm, Robert-Koch St 8, 89081 Ulm, Germany (E-mail: martin.hetzel@medizin.uniulm.de).

J Thorac Cardiovasc Surg 2004;127: $1427-31$

$0022-5223 / \$ 30.00$

Copyright $(\odot) 2004$ by The American Association for Thoracic Surgery

doi:10.1016/j.jtcvs.2003.12.032
Objectives: Endobronchial cryotherapy is an established recanalization method for stenoses of the respiratory tract. However, previous applications of cryotherapy have not been immediately effective, requiring a second clean-up procedure several days later to finally obtain sufficient recanalization. In this study we demonstrate a newly developed cryoprobe allowing recanalization of tumor stenoses during a single intervention.

Methods: In this prospective study flexible bronchoscopy was used for cryorecanalization of 60 patients with high-grade stenoses of the respiratory tract from exophytic tumors. Tumor tissue was frozen on the tip of the probe and subsequently removed from the surrounding respiratory tract tissue through retraction of the probe. Procedures were carried out on sedated, spontaneously breathing, intubated patients.

Results: Fifty (83\%) of 60 patients were successfully or partially successfully treated. Tumor bleeding occurred in 6 patients but was stopped with argon plasma coagulator treatment in all patients not requiring rigid bronchoscopy.

Conclusion: Cryorecanalization with the newly developed cryoprobe permits effective, safe, and inexpensive therapy of endobronchial stenoses of the respiratory tract.

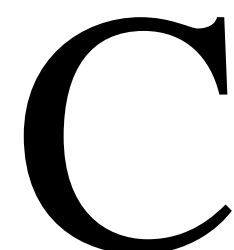

urrently, effective methods for the treatment of stenoses and closures of the respiratory tract caused by exophytic tumors include laser therapy with the Nd-YAG laser, mechanical recanalization (rigid bronchoscopic debulking), photodynamic therapy (PDT), and cryotherapy. ${ }^{1-3}$ The disadvantages of these methods include the necessity of clean-up bronchoscopy to remove tumor necroses or fibrin membranes (PDT and cryotherapy), the necessity of a primary procedure with rigid bronchoscopy in total anesthesia (laser therapy and mechanical recanalization), the lack of immediate effectiveness (cryotherapy and PDT), and high therapeutic costs (PDT) ${ }^{1-3}$ In cryotherapy extreme cold is locally applied to induce tumor necrosis by using a rigid or flexible probe. In a second bronchoscopy 5 to 10 days after the first intervention, necrotic tumor tissue is removed. ${ }^{4}$ This strategy, although effective, does not allow immediate recanalization of stenotic or occluded airways. Thus we have examined the possibility of immediate exophytic tumor tissue extraction after tumor freezing on the tip of a newly developed probe; this procedure is referred to below as cryorecanalization. Initial experimental data from our group showed that commercially available flexible cryoprobes used for cryotherapy are not suitable for cryorecanalization because of their relatively low freezing power and the lack of stability to traction of the gas channel in the probe tip. Because of these limitations, only small pieces of tumor tissue could be extracted when these probes were used; in addition, the gas channel was often dislocated from the probe tip by means of traction, leading to defective probe function. These disadvantages could be 


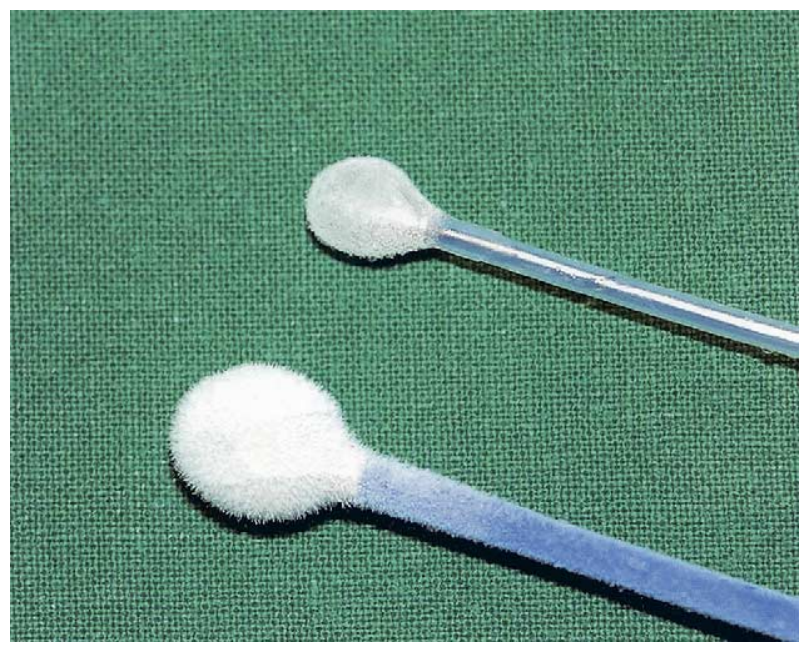

Figure 1. Cryotherapy probe (back) and cryorecanalization probe (front): formation of an ice ball after 1 minute of freezing in a water bath. The freezing power of the cryorecanalization probe is greater.

eliminated with a cryoprobe specifically designed for recanalization. The present study sought to investigate the effectiveness of cryorecanalization with such a newly developed probe in patients with respiratory tract stenosis caused by exophytic tumors.

\section{Materials and Methods \\ Cryoprobe}

The probe used for cryorecanalization was $78 \mathrm{~cm}$ in length and 2.3 $\mathrm{mm}$ in diameter. Differences from the probe used for cryotherapy include stable attachment of the central gas channel in the probe tip, resulting in greater stability to traction $(50 \mathrm{~N})$. In addition, greater freezing power (Figure 1) was achieved by extending the probe's surface area. A hydrophilic coat facilitates the exact guidance of the probe in the working channel of the bronchoscope. In accordance with the Joule-Thompson principle, the probe tip is cooled by means of gas decompression in the probe head. ${ }^{5}$ The instrument we used (Erbokryo; ERBE Medizintechnik GmbH, Tübingen, Germany) uses nitrous oxide at an exit temperature of $-89.5^{\circ} \mathrm{C}$ at the tip of the probe. The probe is a closed system that can be resterilized and reused. Application for registration of the probe has been made.

\section{Patients}

Indications for cryorecanalization were based on the following findings:

- high-grade stenosis in the area of the central respiratory tracts from exophytic tumors with clinical symptoms (dyspnea or post-obstructive pneumonia) and

- stenosis-closure of a lobe or segment of a bronchus with postobstructive pneumonia.

An airway narrowing that could not be passed with an Olympus BF160P bronchoscope (outer diameter, $4.9 \mathrm{~mm}$ ) despite pressure exerted on the bronchoscope was defined as a high-grade stenosis.
TABLE 1. Cause of the stenoses

\begin{tabular}{lc}
\hline Cause of the stenosis of the respiratory tract & $\begin{array}{c}\text { No. of } \\
\text { patients }\end{array}$ \\
\hline Bronchial carcinoma & 51 \\
Metastases of a renal cell carcinoma & 2 \\
Metastases of carcinoma of the colon & 2 \\
Bronchial carcinoid & 1 \\
Benign granulations on tracheal stents & 3 \\
Malignant lymphoma & 1 \\
\hline
\end{tabular}

TABLE 2. Anatomic position of the stenoses and outcome of cryorecanalization

\begin{tabular}{|c|c|}
\hline $\begin{array}{l}\text { Anatomic position of the } \\
\text { stenosis }\end{array}$ & $\begin{array}{c}\text { No. of patients with } \\
\text { successful/partially } \\
\text { successful/unsuccessful } \\
\text { outcome }\end{array}$ \\
\hline Trachea & $5 / 1 / 0$ \\
\hline Main bronchus & $17 / 6 / 4$ \\
\hline Upper lobe bronchus & $4 / 1 / 2$ \\
\hline Lower lobe bronchus & $6 / 3 / 1$ \\
\hline Middle lobe bronchus & $1 / 0 / 1$ \\
\hline Intermediate bronchus & $4 / 2 / 2$ \\
\hline
\end{tabular}

Patients with airway stenoses caused by extrinsic compression were not included. A thrombocyte count of greater than $100 \mathrm{G} / \mathrm{L}$ and normal plasma clotting were prerequisites for the performance of cryorecanalization. From January 2002 through May 2003, all patients who presented at our department with these criteria were included in the study. Cryorecanalization was performed in 60 patients aged 19 to 81 years. The causes and anatomic positions of the endobronchial lesions in the group of patients are given in Tables 1 and 2, respectively. Of 60 patients, 23 had complete bronchial obstruction, whereas 37 had high-grade airway stenoses. Of 23 patients, 17 had segmental or lobar atelectasis. All patients were fully informed of possible complications and then gave their written consent to treatment. The study was approved by the local ethics committee.

\section{Cryorecanalization}

The cryorecanalization procedure is shown in Figure 2. After inhalation of local anesthetic, patients were intubated with an 8.5-mm Bronchoflex tube (Rüsch $\mathrm{GmbH})$. Oxygen (6-12 L/min) was continuously insufflated through this tube. The procedure was performed during sedation with propofol (200-2400 mg), with spontaneous respiration. Patients were monitored by means of continuous pulse oximetry, electrocardiography, and measurement of blood pressure every 2 minutes. Eight patients temporarily required a dopamine infusion for arterial hypotension during the intervention.

Cryorecanalization was performed by means of flexible bronchoscopy with an Olympus BF-1T160. The cryoprobe, guided through the working channel of the bronchoscope, was placed inside the tumor $(1-2 \mathrm{~cm})$. The probe tip was then cooled for 5 to 20 seconds. In most cases, freezing of the tumor tissue was 

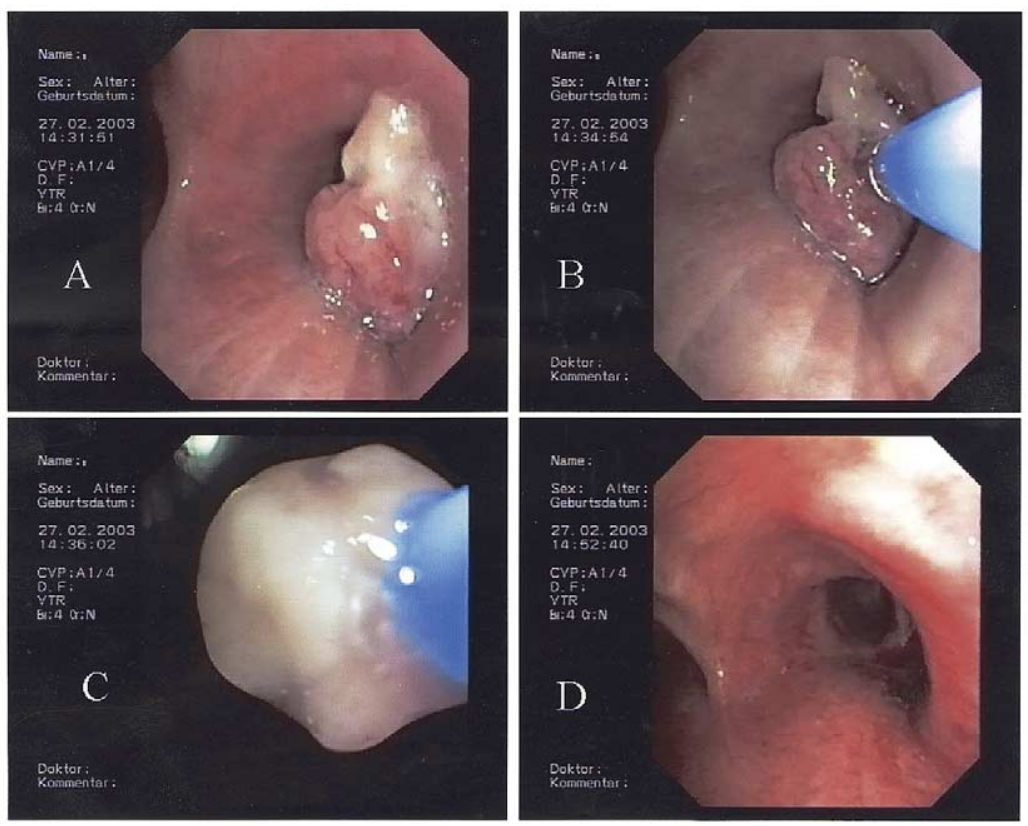

Figure 2. A, Exophytic tumor (colon carcinoma metastasis) in the right main bronchus. B, Freezing the tumor with the cryorecanalization probe. C, Extraction of tumor tissue. D, Right main bronchus after cryorecanalization.

endoscopically visible. Control of the ice front allowed good assessment of the local extension of tissue freezing. Before the ice front reached the healthy bronchial wall, frozen tumor tissue was extracted from its surrounding tissue by pulling strongly on the probe. Together with the bronchoscope, the tissue stuck to the tip of the cryoprobe was pulled out of the respiratory tract. Frozen tumor tissue was then released from the probe tip by thawing in a water bath. The aim of the intervention was to repeat this procedure until no relevant stenosis from exophytic portions of the tumor remained. Mild bleeding around the site of removal of the tumor was treated with topical application of adrenalin. More intense bleeding was treated with argon plasma coagulation.

\section{Evaluation of the Recanalization}

Three different degrees of therapeutic success were distinguished:

- Successful: no residual stenosis detectable by means of endoscopy (reference segment, bronchial segment without pathologic changes that lies immediately distal to the stenosis);

- Partially successful: residual stenosis evident in endoscopy but stenosis passable with an Olympus BF-1T160 (optical density, $6 \mathrm{~mm}$ ) without pressure; and

- Unsuccessful: persistent nonpassable stenosis or closure.

\section{Results}

Cryorecanalization Allows Immediate Recanalization of Respiratory Tract Stenoses

Sixty patients were treated. Complete recanalization was achieved in $37(61 \%)$ patients. Thirteen (22\%) patients exhibited residual stenoses that were easily passable with a bronchoscope. Their treatment was rated as partially suc- cessful. In $10(17 \%)$ patients treatment was unsuccessful. Tumor closures in these patients turned out to be too extended. No connection with the distal respiratory tract was achieved, even though large quantities of tumor tissue were extracted. The procedure lasted for between 9 and $81 \mathrm{~min}-$ utes (41 \pm 16 minutes). On average, 13 (range, 3-31) applications of the cryoprobe were required during one intervention. Treatment success in our patients was independent of the tumor cause and independent of the localization of the stenosis.

Of 57 patients with malignant airway disease, 14 presented again with symptoms of recurrent airway obstruction. The time between the interventions ranged from 10 to 24 weeks (18 \pm 4 weeks; median time, 16 weeks). Thirty patients died during follow-up after 36 weeks on average (range, 6-67 weeks). Thirteen patients are still alive (mean time of follow-up, 35 weeks; range, 24-71 weeks) without any symptoms from airway stenosis. Follow-up of these patients is still going on. However, all but 4 patients with malignant airway stenoses received additional chemotherapy, radiation therapy, or both.

\section{Cryorecanalization is a Safe Procedure}

None of the patients died during the procedure. Rigid bronchoscopy to treat bleeding or other complications was not needed in any of the patients. No patient had to be started on mechanical ventilation after the procedure. After cryorecanalization, 7 patients were subjected to intensive medical monitoring for 12 hours because of high doses of propofol. 
Fifty-four patients exhibited light bleeding, which stopped spontaneously within a couple of minutes. Six patients had more intense bleeding (amount of blood loss, 100-300 mL) at the site of tumor adhesion after removal of tumor tissue. In all of these patients, bleeding could be controlled with suction with the flexible bronchoscope and was stopped in all patients by using an argon plasma beamer and flexible technology.

\section{Discussion}

\section{Effectiveness of Cryorecanalization}

In contrast to cryotherapy, cryorecanalization with a newly developed probe is immediately effective in recanalization of airway stenoses. This novel technique does not require clean-up bronchoscopy several days after the cold treatment to remove tumor necroses. The frozen tumor tissue sticking to the cooled probe tip is extracted immediately during the procedure. Therefore this method is also suitable for patients with acute symptoms caused by airway stenosis.

In $83 \%$ of our patients, cryorecanalization was partially or completely successful. In those patients in whom no recanalization could be achieved, tumor stenosis turned out to be too extensive and thus did not allow connection with the distal respiratory tract. However, high-grade airway strictures should be considered for cryorecanalization only when distal airway patency is preserved. As a suitable method for better patient selection, we suggest virtual bronchoscopy if available ${ }^{6}$ or bronchography. With these pretreatment procedures, probably the majority of unsuccessful interventions in our study group could have been avoided.

Success rates of Nd-YAG laser therapy, which is the most frequently used and best evaluated method for the immediate management of endoluminal airway obstructions, range from $50 \%$ to $90 \%$ depending on the location of the lesion. ${ }^{7}$ In our study cryorecanalization was successful in $83 \%$ of the treated patients, with no difference between tracheal or main stem bronchi and lobar bronchi lesions, respectively.

\section{Safety of Cryorecanalization}

After extraction of frozen tumor tissue, endobronchial bleeding requiring argon plasma beam coagulation occurred in 6 of 60 patients. Cold causes vasoconstriction and capillary microthromboses in the border area between frozen and nonfrozen tissue. ${ }^{5}$ These effects likely contribute to the low rate of bleeding complications with this method. Visual control of the ice front is possible while the tissue is being frozen, and this allows a relatively reliable assessment of the depth of action. The rate of advance of the ice front decreases after longer times of freezing, providing an additional safeguard against accidental freezing of healthy tissue. Accidental freezing of larger rings of cartilage is recognizable because the probe cannot be removed from the bronchus, even if it is very strongly pulled. The probe can then readily be released from the bronchial cartilage at the end of the freezing process. The water content of cartilage tissue is low, and this is probably the reason that cold causes little cartilage necrosis in contrast to thermal energy. ${ }^{2}$ Furthermore, in contrast to laser therapy, with its high thermal energy release, cryorecanalization will be applicable in patients with coated airway stents and can be done at high oxygen concentrations without increased risk.

Cryorecanalization works through conversion of fluidrich and vascularized tumor tissue close to the tip of the probe into an adherent homogeneous ice ball and subsequent extraction of the frozen mass. The transition zone from the tumor ice ball to the healthy airway wall, with its unvascularized cartilage rings, represents an area of inhomogeneous ice formation. ${ }^{8}$ This zone is believed to be the breaking area when traction is exerted on the probe, thus preserving the healthy airway wall. Hence this mechanism is likely to contribute to the safety of cryorecanalization.

Six patients exhibited more intense bleeding (amount of blood loss, $<300 \mathrm{~mL}$ in each patient). In all of these patients, bleeding could be controlled with the suction of the flexible bronchoscope and was stopped by argon plasma beam coagulation. We assume, although we cannot prove, the bleeding in these 6 patients would have stopped on its own if we had waited longer before using the argon plasma coagulator. Recent preliminary data from our group suggest that the physiologic clotting system is indeed sufficient to stop diffuse bleeding after tissue extraction. In conclusion, we do not recommend argon plasma beam coagulation to be included in the armamentarium required for cryorecanalization, but we do recommend that rigid bronchoscopy be made immediately available for potential complications with this new method.

\section{Conclusion}

In contrast to PDT and cryotherapy, cryorecanalization is immediately effective in the treatment of respiratory tract stenoses caused by exophytic tumors. Also, cryorecanalization does not require clean-up bronchoscopy. In addition, our experience shows that cryorecanalization can be performed with flexible technology. Moreover, cryorecanalization is the most inexpensive of all recanalization methods because the total therapeutic system can be purchased for around $7000 €$. The rate of complications with this method is low, most likely because of the relative bioselectivity of the action of low temperatures on vascularized tumor tissue and the induction of vascular thromboses. ${ }^{8}$ On the basis of our current experience with 60 patients, rigid bronchoscopy is not necessary but should be kept immediately available for potential complications with this new method. To our knowledge, this is the first feasibility study with cryorecanalization in patients with respiratory tract stenoses caused by exophytic tumors. Still, future studies are needed to 
compare this novel technique with other recanalization methods.

In summary, cryorecanalization treatment with the newly developed probe is a feasible technique and might offer many advantages in the interventional therapy of stenosing exophytic tumors of the respiratory tract: use of flexible technology, immediate effectiveness, low risk of complications, and low costs in comparison with those of other recanalization methods.

The authors thank ERBE Medizintechnik GmbH, Tübingen, Germany, for their contribution to this study.

\section{References}

1. Seijo LM, Sterman DH. Interventional pulmonology. $N$ Engl J Med. 2001;344(10):740-9.
2. Dumon JF, Reboud E, Garbe L, Aucomte F, Meric B. Treatment of tracheobronchial lesions by laser photoresection. Chest. 1982;81:27884.

3. McCaughan JS Jr. Survival after photodynamic therapy to non-pulmonary metastatic endobronchial tumors. Lasers Surg Med. 1999;24:194201.

4. Mathur PN, Wolf KM, Busk MF, Briete WM, Datzman M. Fiberoptic bronchoscopic cryotherapy in the management of tracheobronchial obstruction. Chest. 1996;110:718-23.

5. Maiwand MO, Homasson JP. Cryotherapy for tracheobronchial disorders. Clin Chest Med. 1995;16:427-43.

6. Liewald F, Lang G, Fleiter T, Sokiranski R, Halter G, Orend KH. Comparison of virtual and fiberoptic bronchoscopy. Thorac Cardiovasc Surg. 1998;46(6):361-4.

7. Cavaliere S, Venuta F, Foccoli P, Toninelli C, La Face B. Endoscopic treatment of malignant airway obstructions in 2008 patients. Chest. 1996;110:1536-42.

8. Rubinski $\mathrm{B}$. The process of freezing and the mechanism of damage during cryosurgery. In: Homasson JP, Bell JB, editors. Cryotherapy in chest medicine. 1st ed. Paris: Springer; 1992. p. 7-17. 